\title{
Antimicrobial resistance in bacterial pathogens among hospitalized children with community acquired lower respiratory tract infections in Dongguan, China (2011- 2016)
}

Xiaoguang $\mathrm{He}^{1,2,3 \dagger}$, Mingyu Xie $\mathrm{ie}^{1,2,3 \dagger}$, Siping $\mathrm{Li}^{1,2,3}$, Junqin $\mathrm{Ye}^{1,2,3}$, Qi Peng ${ }^{1,2,3}$, Qiang Ma ${ }^{1,2,3}$, Xiaomei Lu ${ }^{1,2,3^{*}}$ and Baimao Zhong ${ }^{1,2,3^{*}}$

\begin{abstract}
Background: Bacterial pathogens are a major cause of childhood community acquired lower respiratory tract infections (CA-LRTIS), and few data described the impact of antimicrobial resistance on children with CA-LRTIs. This study aims to investigate the antimicrobial resistance in common bacterial agents among hospitalized children with CA-LRTIs between 2011 and 2016 in Dongguan, China.

Methods: Sputum samples were collected from hospitalized children (0-5 years old) with CA-LRTIs in Dongguan Children's Hospital. Bacterial pathogens were detected using traditional culture methods, and disc diffusion tests were used to determine antibiotic resistance.

Results: Among the 2360 samples analyzed, 342 (14.5\%) were positive for bacterial infection. The most prevalent pathogen was MSSA (2.3\%), followed by MRSA (1.5\%), E. coli (1.7\%), E. coli ESBLs (1.2\%), K. pneumonia ESBLs (1.5\%), K. pneumonia (1.4\%) and S. pneumonia (1.3\%). Of the hospitalized patients with bacteria causing of CA-LRTIs, 90.1\% were less than 1-year-old. MSSA and MRSA were more commonly isolated in infants less than 3 months. E. coli, $K$. pneumonia and K. pneumonia ESBLS were more common bacteria causing CA-LRTIs in infants less than 1 month. Resistance levels to penicillins, fluoroquinolones, macrolides, cephalosporins, carbapenems and vancomycin varied in different bacteria.
\end{abstract}

Conclusions: S. aureus, E coli and K. pneumonia were the common bacterial isolates recovered from chidren with CA-LTRIs during 2011-2015. Age group of under 1 year old was at a high risk of bacterial infections. Many isolates showed antibiotic resistance level was associated with antibiotic usage in clinic. Increasing surveillance of antibiotic resistance is urgently needed and develops better strategies to cure the antibiotic abuse in China.

\footnotetext{
*Correspondence: Ixm020@126.com; zbomao@163.com

${ }^{\dagger}$ Equal contributors

${ }^{1}$ Department of Pediatric Respiratory Medicine, Dongguan Children's

Hospital, Xihu Third Road NO. 68, Dongguan, Guangdong 523325, China

Full list of author information is available at the end of the article
} 


\section{Background}

Community acquired lower respiratory tract infections (CA-LRTIs) continues to be one of the most frequent infectious diseases causes of hospitalization and death worldwide, especially in children less than five years old [1-4]. Although respiratory viruses,such as respiratory syncytial virus, adenovirus and human metapneumovirus are significantly implicated in LRTIs at present, bacterial pathogens remain a major cause of CA-LRTIs in children, particularly in developing areas [5-7]. Among several previous Chinese studies, Qin et al. [8] found Streptococcus pneumoniae and Haemophilus influenzae were the predominant pathogens in Nanjing. Pei et al. [9] reported S. pneumoniae and Escherichia coli were the major bacterial agents in Xinxiang. Peng et al. [10] found S. pneumoniae and Hemophilus parainfluenzae were the main pathogens in Chongqing. The attributable fractions of each of these pathogens varied largely depending on geographical locations. However, limited information about bacterial pathogens trigging CA-LRTIs were reported in Dongguan, Southern China.

Antibiotic resistance is considered to be a worldwide problem, and unwise use of antibiotics has been recognized as a key contributor to the increasing rates of resistance [11]. Therefore, clinical practice typically uses empirical antibiotic selection to target the most likely pathogens based on antibiotic sensitivity data [12]. The antibiotic resistance associated with CA-LRTIs varies significantly depends on geographical locations and investigated populations [13, 14]. Therefore, it is not adequate to simply copy the existing guidelines from other countries, which may be inappropriate and lead to serious problems in clinical practice. For example, the incidence of aminoglycosides and quinolones resistant-MRSA is relatively high in the USA [15] and Thailand [16]. Penicillin-resistant $S$. pneumoniae is also relatively high in USA [17] and Southeast Asia [18]. Moreover, most studies have described patterns in the resistance of bacterial pathogens among adults with respiratory tract infections, these patterns may occur differently in adults and children [14, 19]. Dongguan is an industrial city in southern China, which has more than 10 million of people. Limited information regarding antimicrobial resistance patterns in Dongguan is available, and it is necessary to clarify the epidemiology and antibiotic resistance in this local area. The objectives of our study were to identify antimicrobial resistance in microorganisms in children diagnosed with CA-LRTIs in Dongguan, China; to provide guidance to local clinical pediatricians regarding the choice of appropriate antibiotic therapy and decrease the incidence of antimicrobial resistance.

\section{Methods}

\section{Study population}

This retrospective study was conducted at the Department of Pediatric Infectious Disease in Dongguan Children's Hospital, which is a tertiary care center with over 300 beds, including Department of Pediatric Intensive Care Unit, Department of Neonatal Intensive Care Unit, Department of Pediatric Surgery, Department of Pediatric Orthopaedic and several key departments of common pediatric internal medicine, the daily outpatient visits are more than 1000 .

During the period from March 2011 to June 2016, the hospitalized patients less than 18 years old displaying symptoms of CA-LRTIs were enrolled in this study if they met three inclusion criteria: 1) one or more respiratory symptoms, including cough, expectoration, dyspnea, pleuritic pain, or/and fever; 2) radiographic findings indicating evidence of pneumonia/bronchitis, such as a chest X-ray or computed tomography (CT) scan interpreted by an attending radiologist as showing pulmonary consolidation, opacity, or infiltrate; and 3) children suffering lower respiratory tract infections before admission. The exclusion criteria are: 1) Immunodeficiency disease; and 2) known Mycoplasma pneumoniae or Chlamydia pneumoniae infections, in that Mycoplasma pneumoniae antibodies were detected using Passive Particle Agglutination (SERODIA ${ }^{\oplus}$-MYCO II, Japan), and lgG/M antibody to Chlamydia pneumoniae was detected using Colloidal (China) in our hospital.

\section{Microbiological methods}

All the pediatric patients involved were asked to supply single sputum samples from lower respiratory tract for 1-2 ml before initiation of antibiotic therapy. Regrettably, blood samples were not obtained in the retrospective study. As obtaining meaningful sputum specimens were extremely difficult in babies, the clinical specimens in our research from lower respiratory tract were collected using sterility sputum aspirating tubes by trained personnel following standard operating procedures. All samples had been transported to the laboratory for processing and analysis under the conditions of $\leq 2 \mathrm{~h}$ and $4{ }^{\circ} \mathrm{C}$. Each specimen must be in accordance with the microscopic quality criteria: squamous epithelial cells $(\mathrm{SEC})<10$ and white blood cells $(\mathrm{WBC})>25$, or $\mathrm{SEC} / \mathrm{WBC}<1: 2.5$. 10ul sputum sample was streaked on MacConkey, blood and chocolate agar, respectively. MacConkey and blood agar were cultured under aerobic conditions in $35 \pm 2{ }^{\circ} \mathrm{C}$ for $16-24 \mathrm{~h}$. The chocolate agar was cultured under aerobic conditions in a $5 \% \mathrm{CO} 2$ at $35 \pm 2{ }^{\circ} \mathrm{C}$ for $16-$ $24 \mathrm{~h}$ according to the guidance. Suspicious colonies were then subcultured on Tryptic Soy Agar using streak plat method for purification, preserved on 
semi-solid agar slants and stored in the refrigerator (with freezing temperature of about $4{ }^{\circ} \mathrm{C}$ ) for subsequent analysis. The bacteria were identified by ATB Expression (BioMerieux, France) that is automatic detection machine. Pseudomonas aeruginosa ACTT 27853, Escherichia coli ATCC25922 and Staphylococcus aureus ATCC25923 were the reference strains of positive control. All the operations were in accordance with the manual.

Antibacterial susceptibility testing was performed using a semiquantitative agar diffusion test. We adjusted the turbidity of clinical cultures with sterile saline or broth to achieve a turbidity equivalent to a 0.5 McFarland standard, and then spread on MuellerHinton (MH) agar plate (Oxoid, USA). Dics should be applied to the $\mathrm{MH}$ agar plate within $15 \mathrm{~min}$ of inoculation. After incubation measure the diameters of zones of complete inhibition to the nearest $\mathrm{mm}$. Zone margins should be read as the area showing no obvious growth detected by the unaided eye. Isolates were categorized as susceptible, intermediate, or resistant to each antibiotic according to CLSI classifications. Extended-spectrum beta-lactamases production, methicillin-sensitive Staphylococcus aureus and carbapenem-resistant Pseudomonas aeruginosa were identified using CLSI criteria (CLSI: http://clsi.org) (The 2011-2016 year of CLSI guidelines, the CLSI guidelines was renewed annually, and carried out according to the latest guidance.). Quality control was carried out using Escherichia coli ATCC25922, Staphylococcus aureus ATCC 29213, Klebsiella pneumonia ACTT 700603, Pseudomonas aeruginosa ACTT 27853, S. pneumonia 49,619, Enterobacter ATCC 35218. Our laboratory is under the supervision of Guangdong Clinical Test Center (a professional QS control organization in Guangdong province), the microbiological laboratory quality assurance was carried out three times a year with accordance to CLSI guidelines (CLSI: http://clsi.org).

\section{Data collection}

Basic demographic data, including age, gender, date of hospitalization and investigation results, were recorded at bedside by a clinician. All laboratory data were recorded in the hospital computer data system.

\section{Statistical analysis}

Statistical analysis was performed using the statistical software of SPSS (version 22; SPSS Inc., Chicago, IL, USA). Chi-squared and Fisher's exact tests were used to compare categorical data. All tests were two-tailed, and a $p$ value $<0.005$ was considered to be statistically significant (adjusting test level "a' $=0.005$ ").

\section{Results}

\section{Detection and distribution of bacteria}

From March 6, 2011 to June 5, 2016, a total of 2360 hospitalized pediatric patients met the inclusion criteria, including 1245 males and 1115 females; the mean age of these patients was 7.4 months (standard deviation: 10.3 months; range $0-5$ years). Of the patients with CA-LRTIs, the majority were younger than 1 year-old (83.1\%). 342(14.5\%) bacteria were indentified, and the most frequent pathogen was Methicillin Sensitive Staphylococcus aureus (MSSA) (2.3\%), followed by Escherichia coli (E. coli) (1.7\%), Methicillin Resistant Staphylococcus aureus (MRSA) (1.5\%), Klebsiella pneumonia Extended Spectyum $\beta$-Lactamase (K. pneumonia ESBLs) (1.5\%), K. pneumonia (1.4\%, Streptococcus pneumoniae (S. pneumonia) (1.3\%), Escherichia coli Extended Spectyum $\beta$-Lactamase (E. coli ESBLs) (1.2\%), Pseudomonas aeruginosa ( $P$. aeruginosa) $(1.0 \%)$, and other pathogens (2.7\%) (Fig. 1).

In this study, all hospitalized patients with CA-LTRIs were divided into five age groups (Table 1). In the study, we found that infants (less than 1 month) were more common population with bacteria agents causing CALRTIs.Our results also showed that MSSA and MRSA were more commonly isolated in infants less than 3 months than that of other three age groups $(\mathrm{P}<0.005)$. E. coli, K. pneumonia and $K$. pneumonia ESBLs were more common bacteria causing CA-LRTIs in infants less than 1 month than that of other four age groups $(\mathrm{P}<0.005)$. However, our results indicated that $P$. aeruginosa, E. coli ESBLs and S. pneumonia infections had no significant difference in the five age groups.

\section{Antimicrobial resistance}

Table 2 illustrats the antimicrobial resistance of the most frequent microorganisms to penicillins, fluoroquinolones, macrolides, cephalosporin, carbapenems and vancomycin during 2011-2016. MSSA showed high resistance level of 81.5\% to penicillin. All MSSA were sensitive to cefazolin in the study. Tested MRSA showed higher resistance to macrolides than MSSA, including erythromycin ( $81.8 \%$ vs. $29.6 \%)$, azithromycin ( $83.3 \%$ vs. $22.2 \%)$ and clarithromycin (82.8\% vs. $34.5 \%)$. Noteworthly, MSSA exhibited higher resistance level to ofloxacin than MRSA (61.2\% vs. 6.5\%). Isolates of MSSA and MRSA exhibited similarity low resistance rates to gentamicin (4.2\% and 9.4\%, respectively) and co-trimoxazole (4.0\% and $13.3 \%$, respectively).

E. coli exhibited high resistance to piperacillin and ampicillin, but relative low resistance to other antibiotics included ofloxacin, gentamicin and cephalosporins. Similarly, E. coli ESBLs had low resistance to ofloxacin and gentamicin, but only it had low resistance to ceftazidime and cefepime in the study. 


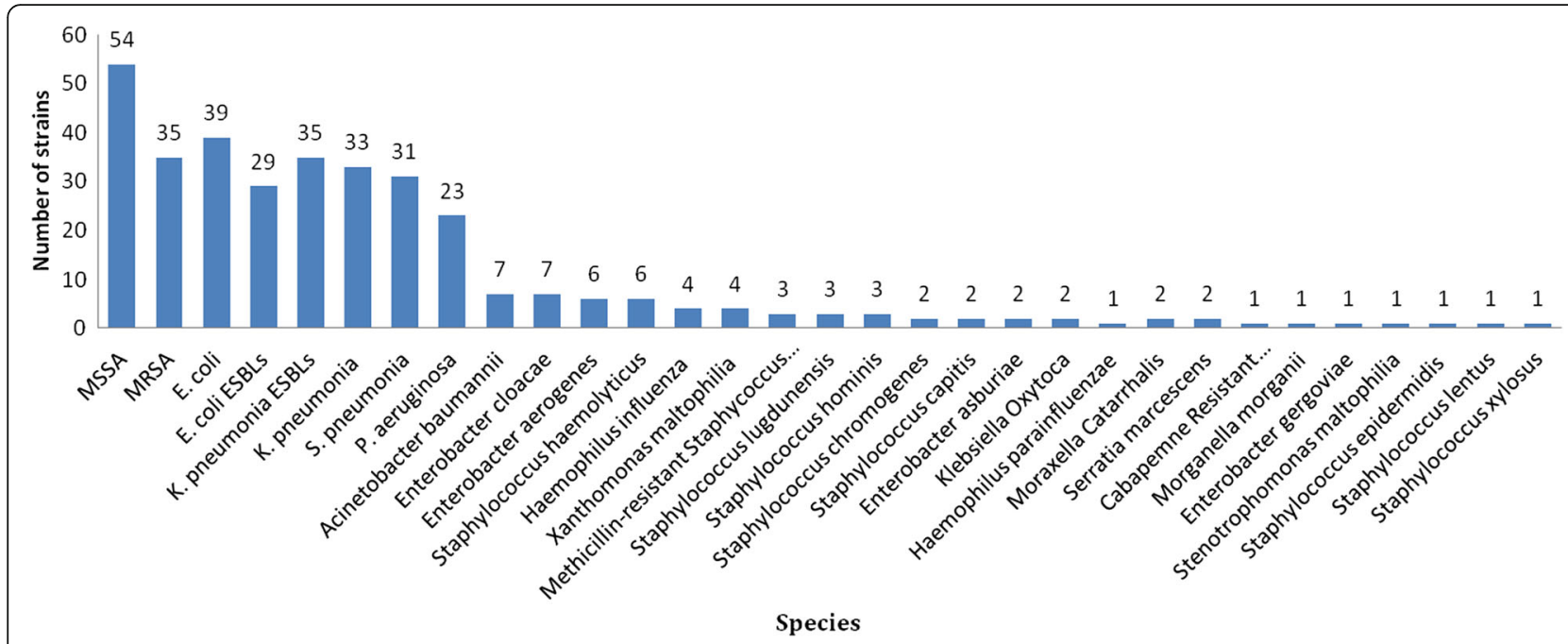

Fig. 1242 bacterial pathogens from 2360children with CA-LRTIs

In the present study, K. pneumonia and K. pneumonia ESBLs isolates showed similar resistance level to ofloxacin $(3.7 \%$ vs. $3.6 \%)$, gentamicin $(13.8 \%$ vs. $13.8 \%)$ and cotrimoxazole (75.0\% vs. $82.1 \%)$. K. pneumonia displayed low resistance to cephalosporins, but $K$. pneumonia ESBLs exhibited high resistance levels to cephalosporins except for cefepime (44.1\%). However, our results showed $24.2 \%$ of piperacillin against $K$. pneumonia.

S. pneumonia isolates had a resistant rate of $29.0 \%$ to penicillin, and a high resistance level of macrolides, including erythromycin (96.7\%) and clarithromycin (93.3\%). However, our study indicated that S. pneumonia isolates had a low resistance level to cephalosporins, such as ceftriaxone (7.1\%), cefotaxime (10.0\%) and cefepime (4.2\%).

Our results showed $21.7 \%$ of $P$. aeruginosa were resistant to piperacillin, and had low resistance levels of ceftazidime (13.6\%) and cefepime (4.3\%) in the study. Our study also exhibited a low resistant rate to other antibiotics, including ofloxaxin (10.0\%) and gentamicin (13.6\%).

Additionally, we found no bacterial pathogen was resistant to vancomycin and imipenem in the present study.

\section{Discussion}

CA-LRTIs in infants and children continue to be a significant problem worldwide. In recent years, difficulties related to CA-LRTIs treatment in children have greatly increased because of the emergence of resistance to the most widely used antibiotics against some of the bacterial pathogens involved in the development of the disease $[14,20]$. Our results showed that MSSA was the most prevalence bacterial pathogen in children with CA-LRTIs, especially in infants less than 1 month. However, it was not in agreement with various studies worldwide that showed S. pneumonia to be the most common bacterial agent in children with CA-LRTIs, such as Finland [15], Japan [21] and other cities in mainland China [8-10]. S. aureus nasal/nasopharyngeal colonization was common in the less than 6 months, and the positive results might be influenced by specimen from the upper respiratory tract. Our result showed that which bacterial pathogen causing CA-LRTIs was associated with age. However, we need large data to confirm the distribution characteristics among the age groups in the future.

Previous papers ascertained the MRSA cause of CALRTIs becoming the major worldwide problem [22]. The resistance rate of MRSA to erythromycin was $81.8 \%$. From 2000 to 2005, Chen et al. [22] found 97\% MRSA were resistant to erythromycin in Taiwan. Chen et al. [23] found all the MRSA from infant were resistant to erythromycin during 2008-2009 in Wuhan, China. The decreasing resistance of erythromycin against MRSA may be due to more normative using antibiotics comparing with that in past. MRSA showed resistance rates to ofloxacin (6.5\%), gentamicin (9.4\%) and co-trimoxazole (13.3\%), which was lower than that in USA (ofloxacin of 45\%) [15] and Trinidad (gentamicin of 96.8\%,co-trimoxazole of $93.1 \%$ ) [16]. It was associated with limited antibacterial exposure, prescribing and availability in pediatrics in China, in that aminoglycosides and quinolones were inappropriate therapy for pediatric patients [24]. Aminoglycosides might cause hearing loss and quinolones might casuse achondroplasia in children, thus, the two antibiotics were used with caution in China. In early 1990s, the national guidelines were consistent in recommending a macrolide together with a $\beta$ - 


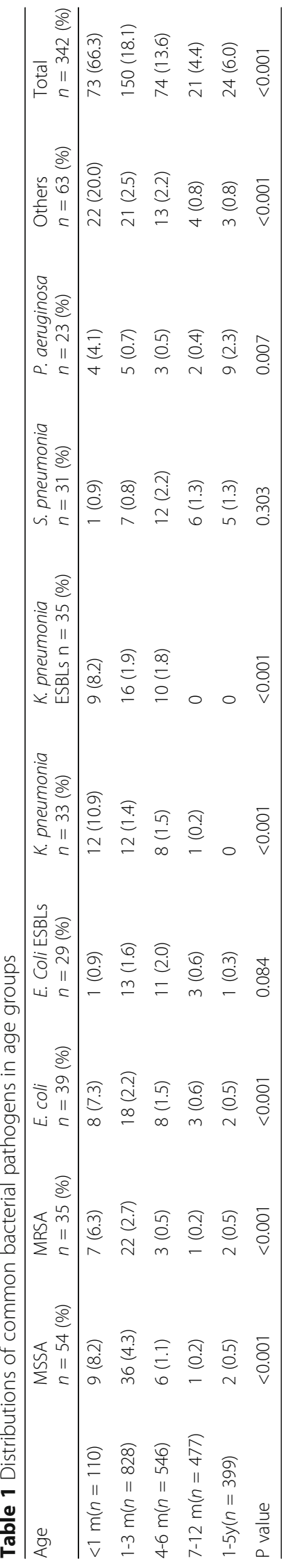




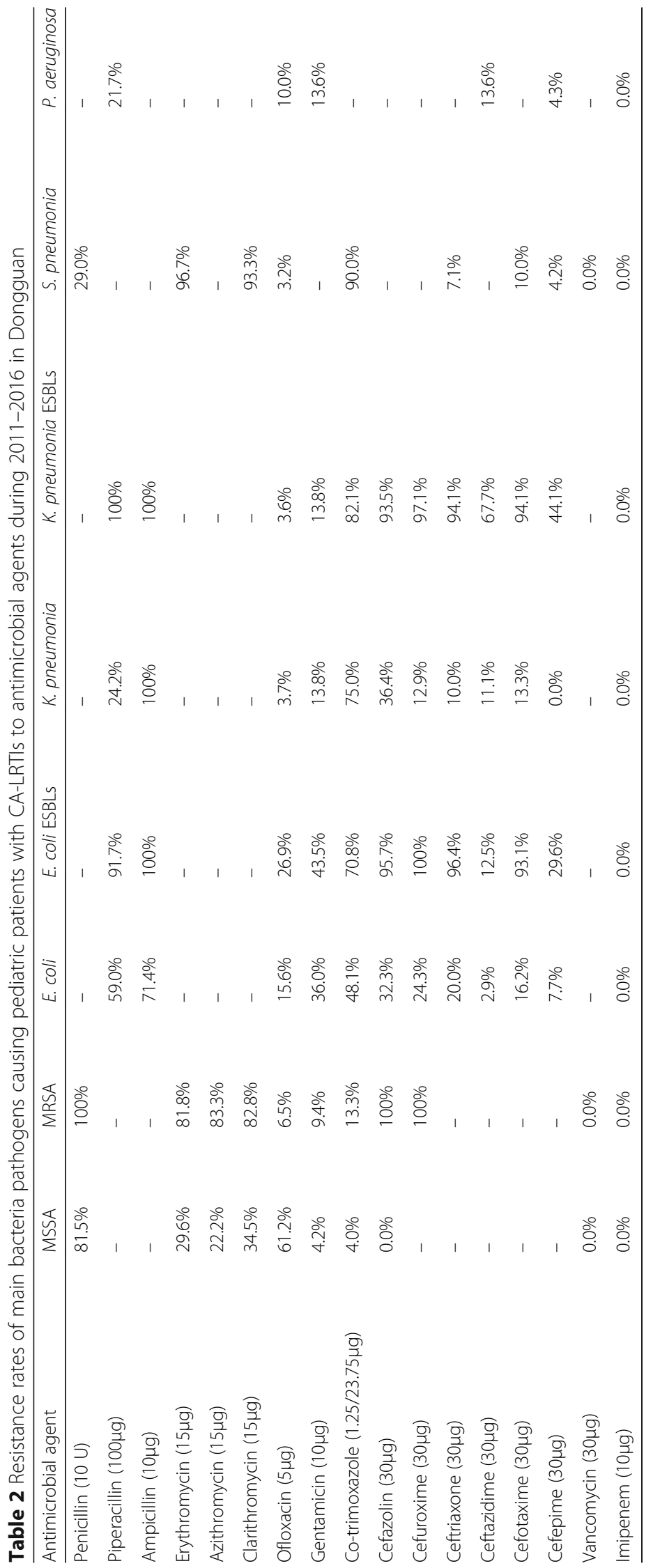


lactam in adults with severe CA-LRTIs [25, 26]. Apparently, $\beta$-lactam was insignificant to CA-LRTIs with MRSA infections. Limited available data suggested how such community-acquired infections were managed in routine practices. Local and national susceptibility data should be taken into account when making recommendations for empiric therapy. In other parts of China, resistance levels of MSSA to penicillin were substantial. Chen et al. [23] found equivalence to high resistance of $82.9 \%$ in Wuhan, China. Hou et al. [27] found 92.5\% MSSA were resistant to penicillin in Shenzhen, China. The resistance rate of MSSA to cefazolin was $0.0 \%$, which was in agreement with to that in Shenzhen [27] and Wuhan [23], China. Cefazolin might be recommended the empiric use as first-line treatment to MSSA causing CA-LRTIs in China.

In our study, S. pneumonia isolates had a resistant rate of $29.0 \%$ to penicillin, which was similar to other surveillance programmers last decade, such as in Taiwan (30\%) [22], USA (25\%) [14] and Mexico City (37\%), but was lower than that in HongKong (56\%). With the development of antibiotics, penicillin was rarely used at present in China. Our results showed that high resistance levels to macrolides, including erythromycin $(96.7 \%)$ and clarithromycin (93.3\%). In the early 1990, the US study indicated that CA-S. pneumonia had a resistance rate of $10 \%$ to erythromycin and clarithromycin [28]. From 2002 to 2005, Matute et al. [29] findings showed CA- S. pneumonia had been sensitive to erythromycin and penicillin in León, Nicaragua. Hsueh et al. [30] found 82\% CAS. pneumonia were resistant to erythromycin in Taiwan during 1996-1997. In 2012, Pan [31] et al. also found high resistance to erythromycin (98.5\%) in Shanghai, China. As observed by various resistance levels, high resistance rates are associated with greater exposure to antibiotics in China [32]. Antibiotics should be used judiciously for reducing antibiotic resistance in China. Fluoroquinolones resistance (ofloxacin, 3.2\%) was uncommon among respiratory tract isolates of $S$. pneumonia in the present study, which was in agreement with previous USA report $(0.7 \%)$ [33]. Our study also identified low resistance to ceftriaxone, which was consistent with that in Shanghai $(1 \%-28.2 \%)$ [34, 35]. Generally, the resistance level of $S$. pneumonia against ceftriaxone was consistence with that to cefotaxime. In our study, it might be due to statistical bias.

K. pneumonia and E. coli were the two Gram-negative bacteria most frequently implicated in causing respiratory tract infections in children in this study, and these results were consistent with other reports from China [36]. They also belong to the family enterobacteriaceae and frequently produce extended-spectrum beta-lactamases. It was important to note that we found no K. pneumonia isolate was resistant to cefepime in our study. It might be due to limited isolates. The resistance rate of $E$. coli ELBLs to cotrimoxazole(70.8\%) and gentamicin (43.5\%) were similar to those reported in Saudi Arabia (co-trimoxazole of 71.1\%, gentamicin of 47\%) [37]. The resistance of $K$. pneumonia ELBLs to co-trimoxazole(82.1\%) and gentamicin (13.8\%) were lower than that in Madagascar (co-trimoxazole of $91.3 \%$, gentamicin of 76.1\%) [38]. Our results also showed the low resistance level to ofloxacin (26.9\%) and cefepine (29.6\%). It suggested that these antibiotics were recommended in the clinical treatment. The appropriate drugs against $K$. pneumonia ESBLs were similar with that to $E$. coli ESBLs. But ceftazidime was not recommended empiric use for children with $K$. pneumonia ESBLs infections.

The emergence of carbapenem-resistant $P$. aeruginosa has been an increasing problem in many parts of the world $[39,40]$. However, we found 2 strains of carbapenemresistant $P$. aeruginosa in our study. One report from Taiwan indicated the risk factor of carbapenem-resistant $P$. aeruginosa infections was associated with using fluoroquinolone [41]. It was consistent with the low resistant rate of $P$. aeruginosa to fluoroquinolone (ofloxacin, 9.5\%) in our study. Our results also showed $P$. aeruginosa had low resistant rates for ceftazidime (13.0\%) and cefepime (4.2\%). The resistance levels of P. aeruginosa to ceftazidime and cefepime were lower than that in Nepal [42].

Many studies reported that multidrug resistance bacterial infections represented a growing public health problem in children, especially infant under 1-year-old [43]. Limited children with MDR infections were found in the retrospective study. It might be explained by a view that most of patients were from the regular ward. Generally, the patients from Intensive Care Units (ICUs) or Pediatric Intensive Care Units (PICU) were high risk population with MDR infections [43].

There are mainly 2 limitations in this study: Firstly, we did not collect the blood specimens of the patients with CA-LRTIs during the retrospective study, or the result would be more persuasive. Secondly, mycoplasma pneumoniae and chlamydia pneumoniae infections were also common in the pediatric patients, but patients detected positive for those two pathogens were not enrolled in the study for the two pathogens were identified by other diagnostic methods, and did not detect antibiotic sensitive test.

\section{Conclusions}

S. aureus, E coli and K. pneumonia were the common bacterial isolates recovered from chidren with CA-LTRIs during 2011-2015. Age group of under 1 year old was at a high risk of bacterial infections. Many isolates showed antibiotic resistance level was associated with antibiotic usage in clinic. Increasing surveillance of antibiotic resistance is urgently needed and develops better strategies to cure the antibiotic abuse in China. 


\section{Abbreviations}

A. baumannii: Acinetobacter baumannii; CA-LRTIs: Community acquired lower respiratory tract infections; CLSI: Clinical and Laboratory Standards Institute; $E$. coli: Escherichia coli; ESBLs: Producing extended-spectrum beta-lactamases; $H$. influenza: Haemophilus influenza; K. pneumonia: Klebsiella pneumonia; MRSA: Methicillin-Resistant Staphylococcus aureus; MSSA: Methicillin-sensitive Staphylococcus aureus; P. aeruginosa: Pseudomonas aeruginosa; $S$. aureus: Staphylococcus aureus; S. pneumonia: Streptococcus pneumonia

\section{Acknowledgements}

We are grateful to Dongguan Children's Hospital (Medical Information department), Mr. Wang Qin for his technical assistance in the data collection. Our thanks also goes to the study volunteers for their generous participation.

\section{Funding}

This study was supported in part by grant 2015A030313803 from Natural Science Foundation of Guangdong Province, grant 2,014,108,101,028 and 2,015,108,101,020 from Dongguan Bureau of Science and Technology for the City Key Program of Science and Technology.

\section{Availability of data and materials}

The raw data are stored at the Medical Information department, Dongguan Children's Hospital and will be provided upon request to the corresponding authors.

\section{Authors' contributions}

Study concept and design: XGH, MYX, XML and BMZ. Acquisition of data, analysis and interpretation of data: All authors. Drafting of the manuscript: $X G H, M Y X, X M L$ and BMZ. Experiments performing: SPL, JQY, QP and QM. Statistical analysis: $X G H, M Y X, X M L$ and BMZ. All authors revised the article and all approved the final version.

\section{Ethical approval and consent to participate}

The study was approved by the ethics committee of the Dongguan Children's Hospital and carried out according to the Helsinki Declaration. All guardians gave their consent for the data obtained during their babies' hospitalization to be retrospectively analyzed, and data analyses were carried out using an anonymous database.

\section{Consent for publication}

Not applicable.

\section{Competing interests}

The authors declare that they have no competing interests.

\section{Publisher's Note}

Springer Nature remains neutral with regard to jurisdictional claims in published maps and institutional affiliations.

\section{Author details}

${ }^{1}$ Department of Pediatric Respiratory Medicine, Dongguan Children's Hospital, Xihu Third Road NO. 68, Dongguan, Guangdong 523325, China. 2Department of Pathogenic Microorganism, Dongguan Institute of Pediatrics, Dongguan, Guangdong, China. ${ }^{3}$ Dongguan Key Laboratory of Pediatric Genetic and Infectious Diseases, Dongguan, Guangdong, China.

Received: 14 March 2017 Accepted: 31 August 2017

Published online: 11 September 2017

\section{References}

1. Vink MA, Bootsma MC, Wallinga J. Serial intervals of respiratory infectious diseases: a systematic review and analysis. Am J Epidemiol. 2014;180(9):865-75.

2. Simpson CR, Steiner MF, Cezard G, Bansal N, Fischbacher C, et al. Ethnic variations in morbidity and mortality from lower respiratory tract infections: a retrospective cohort study. J R Soc Med. 2015;108(10):406-17.

3. Beck AF, Florin TA, Campanella S, Shah SS. Geographic variation in hospitalization for lower respiratory tract infections across one county. JAMA Pediatr. 2015;169(9):846-54

4. Sonego M, Pellegrin MC, Becker G, Lazzerini M. Risk factors for mortality from acute lower respiratory infections (ALRI) in children under five years of age in low and middle-income countries: a systematic review and metaanalysis of observational studies. PLoS One. 2015;10(1):e0116380.

5. Osrin D, Vergnano S, Costello A. Serious bacterial infections in newborn infants in developing countries. Curr Opin Infect Dis. 2004;17(3):217-24.

6. Pavia AT. Viral infections of the lower respiratory tract: old viruses, new viruses, and the role of diagnosis. Clinical infectious diseases: an official publication of the Infectious Diseases Society of America. 2011;52(Suppl 4):S284-9.

7. Adegbola RA, DeAntonio R, Hill PC, Roca A, Usuf E, et al. Carriage of Streptococcus Pneumoniae and other respiratory bacterial pathogens in low and lower-middle income countries: a systematic review and metaanalysis. PLoS One. 2014;9(8):e103293.

8. Qin M, Tian M, Xia W, Wang HY, Shi SY, et al. Etiology of communityacquired pneumonia in children. J Chin Pediatr. 2008;26(4):312-5.

9. Pei LH, Guo YQ, Guo YL. Microbiological etiology of community acquired pneumonia in children. J Appl clin Pediatr. 2011:26(22):1470-1.

10. Peng Y, Shu C, Fu Z, Li QB, Liu Z, et al. Pathogen detection of 1613 cases of hospitalized children with community acquired pneumonia. Zhongguo Dang Dai Er Ke Za Zhi. 2015;17(11):1193-9.

11. Ventola CL. The antibiotic resistance crisis: part 1: causes and threats. Pharmacy and Therapeutics. 2015;40(4):277-83.

12. Zhang $X$, Wang $R$, Di X, Liu B, Liu Y. Different microbiological and clinical aspects of lower respiratory tract infections between China and European/ American countries. J Thorac Dis. 2014:6(2):134-42.

13. Ibrahim ME, Bilal NE, Hamid ME. Comparison of phenotypic characteristics and antimicrobial resistance patterns of clinical Escherichia Coli collected from two unrelated geographical areas. Global journal of health science. 2014;6(6):126-35.

14. Felmingham D, Farrell DJ, Reinert RR, Morrissey I. Antibacterial resistance among children with community-acquired respiratory tract infections (PROTEKT 1999-2000). The Journal of infection. 2004:48(1):39-55.

15. McDougal LK, Fosheim GE, Nicholson A, Bulens SN, Limbago BME, et al. Mergence of resistance among USA300 methicillin-resistant Staphylococcus Aureus isolates causing invasive disease in the United States. Antimicrob Agents Chemother. 2010;54(9):3804-11.

16. Orrett FA. The emergence of mupirocin resistance among clinical isolates of methicillin-resistant Staphylococcus Aureus in Trinidad: a first report. Jpn J Infect Dis. 2008;61(2):107-10.

17. Song JH, Lee NY, Ichiyama S, Yoshida R, Hirakata Y, et al. Spread of drugresistant Streptococcus Pneumoniae in Asian countries: Asian network for surveillance of resistant pathogens (ANSORP) study. Clin Infect Dis. 1999;28(6):1206-11.

18. Felmingham D. Comparative antimicrobial susceptibility of respiratory tract pathogens. Chemotherapy. 2004;50(Suppl 1):3-10.

19. Adam HJ, Baxter MR, Davidson RJ, Rubinstein E, Fanella S, et al. Canadian antimicrobial resistance a: comparison of pathogens and their antimicrobial resistance patterns in paediatric, adult and elderly patients in Canadian hospitals. J Antimicrob Chemother. 2013;68(Suppl 1):i31-7.

20. Esposito S, Principi N. Emerging resistance to antibiotics against respiratory bacteria: impact on therapy of community-acquired pneumonia in children. Drug Resist Updat. 2002;5:73-87.

21. Morozumi M, Chiba N, Okada T, Sakata H, Matsubara K, et al. Antibiotic susceptibility in relation to genotype of Streptococcus Pneumoniae Haemophilus influenzae, and mycoplasma pneumoniae responsible for community-acquired pneumonia in children. J Infect Chemother. 2013;19(3): $432-40$

22. Chen CJ, Su LH, Chiu CH, Lin TY, Wong KS, et al. Clinical features and molecular characteristics of invasive community-acquired methicillinresistant Staphylococcus Aureus infections in Taiwanese children. Diagn Microbiol Infect Dis. 2007:59:287-93.

23. Chen HB, Lu XX, Xia W, Sun JM. Analysis of bacterial distribution and drug resistance in induced sputum of infants with lower respiratory tract infection. Appl Clin Pediatr. 2011;26(22):1725-33.

24. Yan SS, Schreckenberger PC, Zheng XT, Nelson NA, Harrington SM, et al. An intrinsic pattern of reduced susceptibility to fluoroquinolones in pediatric isolates of Streptococcus pyogenes. Diagn Microbiol Infect Dis. 2008:62(2):205-9.

25. The British Thoracic Society. Guidelines for the management of communityacquired pneumonia in adults admitted to hospital. Br J Hosp Med. 1993;49: 346-50.

26. Gialdroni Grassi G, Bianchi L. Guidelines for the management of community-acquired pneumonia in adults. Monaldi Arch Chest Dis. 1995:50:21-7. 
27. Hou LY, Zheng YJ, Deng JK, Zhao RZ. Bacterial etiology and antimicrobial resistance patterns of community-acquired pneumonia in hospitalized children in Shenzhen. Chinese Journal of Microecology. 2008;20(6):586-93.

28. Shortridge VD, Doern GV, Brueggemann AB, Beyer JM, Flamm RK. Prevalence of macrolide resistance mechanisms in Streptococcus pneumoniae isolates from a multicenter antibiotic resistance surveillance study conducted in the United States in 1994-1995. Clin Infect Dis. 1999;29: $1186-8$.

29. Matute AJ, Brouwer WP, Hak E, Delgado E, Alonso E, et al. Aetiology and resistance patterns of community-acquired pneumonia in León. Nicaragua Int J Antimicrob Agents. 2006;28(5):423-7.

30. Hsueh PR, Teng $\sqcup$, Lee LN, Yang PC, Ho SW, et al. Extremely high incidence of macrolide and trimethoprimsulfamethoxazole resistance among clinical isolates of Streptococcus pneumoniae in Taiwan. J Clin Microbiol. 1999;37: 893-901.

31. Pan F, Han L, Kong J, Wang C, Qin H, et al. Serotype distribution and antimicrobial resistance of Streptococcus Pneumoniae causing noninvasive diseases in a Children's hospital. Shanghai Braz J Infect Dis. 2015;19(2):141-5.

32. Yu M, Zhao G, Stalsby Lundborg C, Zhu Y, Zhao Q, et al. Knowledge, attitudes, and practices of parents in rural China on the use of antibiotics in children: a cross-sectional study. BMC Infect Dis. 2014;14:112.

33. Jones RN, Fritsche TR, Sader HS. Therapeutic options among broadspectrum beta-lactams for infections caused by levofloxacin-nonsusceptible Streptococcus Pneumoniae. Diagn Microbiol Infect Dis. 2005;52(2):129-33.

34. Pan F, Han L, Huang W, Tang J, Xiao S, et al. Serotype distribution, antimicrobial susceptibility, and molecular epidemiology of Streptococcus Pneumoniae isolated from children in shanghai. China PloS one. 2015;10(11):e0142892.

35. Hu J, Sun X, Huang Z, Wagner AL, Carlson B, et al. Streptococcus Pneumoniae and Haemophilus influenzae type $b$ carriage in Chinese children aged 12-18 months in shanghai. China: a cross-sectional study BMC infectious diseases. 2016;16:149.

36. Zhou L, Yu SJ, Gao W, Yao KH, Shen AD, et al. Serotype distribution and antibiotic resistance of 140 pneumococcal isolates from pediatric patients with upper respiratory infections in Beijing, 2010. Vaccine. 2011;29(44):7704-10.

37. Somily AM, Habib HA, Absar MM, Arshad MZ, Manneh K, et al. ESBLproducing Escherichia Coli and Klebsiella Pneumoniae at a tertiary care hospital in Saudi Arabia. J Infect Dev Ctrie. 2014;8(9):1129-36.

38. Andriatahina T, Randrianirina F, Hariniana ER, Talarmin A, Raobijaona H, et al. Research article high prevalence of fecal carriage of extended-spectrum $\beta$ lactamase-producing Escherichia Coli and Klebsiella Pneumoniae in a pediatric unit in Madagascar. BMC Infect Dis. 2010;10:204.

39. Cho HH, Kwon GC, Kim S, Koo SH. Distribution of pseudomonas-derived Cephalosporinase and Metallo- $\beta$-lactamases in Carbapenem-resistant Pseudomonas Aeruginosa isolates from Korea. J Microbiol Biotechnol. 2015;25(7):1154-62.

40. Logan LK, Gandra S, Mandal S, Klein EY, Levinson J, et al. Multidrug- and Carbapenem-Resistant Pseudomonas aeruginosa in Children, United States, 1999-2012. J Pediatric Infect Dis Soc. 2016;16:pii: piw064.

41. Lin KY, Lauderdale TL, Wang JT, Chang SC. Carbapenem-resistant Pseudomonas Aeruginosa in Taiwan: prevalence, risk factors, and impact on outcome of infections. J Microbiol Immunol Infect. 2016;49(1):52-9.

42. Khan S, Priti S, Ankit S. Bacteria etiological agents causing lower respiratory tract infections and their resistance patterns. Iran Biomed J. 2015;19(4):240-6.

43. Siddiqui NU, Qamar FN, Jurair H, Haque A. Multi-drug resistant gram negative infections and use of intravenous polymyxin B in critically ill children of developing country: retrospective cohort study. BMC Infect Dis. 2014;14:626

\section{Submit your next manuscript to BioMed Central and we will help you at every step:}

- We accept pre-submission inquiries

- Our selector tool helps you to find the most relevant journal

- We provide round the clock customer support

- Convenient online submission

- Thorough peer review

- Inclusion in PubMed and all major indexing services

- Maximum visibility for your research

Submit your manuscript at www.biomedcentral.com/submit 